\title{
Proposal of six new species in the genus Microbacterium and transfer of Flavobacterium marinotypicum ZoBell and Upham to the genus Microbacterium as Microbacterium maritypicum comb. nov.
}

\author{
Mariko Takeuchi and Kazunori Hatano
}

Author for correspondence: Mariko Takeuchi. Tel: +8163006555 . Fax: +8163006814 . e-mail: fvgg0814@mb.infoweb.ne.jp

Institute for Fermentation, Osaka, 17-85, Jusohonmachi 2-chome, Yodogawa-ku, Osaka 532, Japan

\begin{abstract}
Reference strains, including two mis-named organisms, ' Chromobacterium chocolatum' and Flavobacterium marinotypicum, isolates from soil and clinical specimens, all previously recognized as Aureobacterium or Microbacterium, were characterized taxonomically. On the basis of morphological, physiological and chemotaxonomic characteristics, as well as DNA-DNA hybridization data, six new species and one new combination are proposed in the genus Microbacterium : Microbacterium ketosireducens sp. nov. (type strain IFO 14548'), Microbacterium chocolatum sp. nov. (type strain IFO 3758'), Microbacterium aurantiacum sp. nov. (type strain IFO 15234'), Microbacterium hominis sp. nov. (type strain IFO 15708'), Microbacterium thalassium sp. nov. (type strain IFO 16060'), Microbacterium halophilum sp. nov. (type strain IFO 16062') and Microbacterium maritypicum comb. nov. (type strain IFO 15779').
\end{abstract}

Keywords: Microbacterium ketosireducens sp. nov., Microbacterium chocolatum sp. nov., Microbacterium aurantiacum sp. nov., Microbacterium hominis $\mathrm{sp}$. nov., Microbacterium thalassium sp. nov., Microbacterium halophilum sp. nov., Microbacterium maritypicum comb. nov.

\section{INTRODUCTION}

As a result of the unification of the genera Microbacterium and Aureobacterium, 19 species are accommodated in the genus Microbacterium (Takeuchi \& Hatano, 1998). The taxonomic study that led to the unification revealed that 11 reference strains including two mis-named organisms, 'Chromobacterium chocolatum' and Flavobacterium marinotypicum, isolates from soil and clinical specimens, should be accommodated in the redefined genus Microbacterium, although their taxonomic positions in the genus were not certain. All strains had all of the characteristic chemotaxonomic markers of the genus Microbacterium, the presence of D-diamino acid or L-diamino acid (Dornithine or L-lysine), glycine and $\mathrm{N}$-glycolyl residues in the cell walls, isoprenoid quinones MK-11, 12, and/or 13 , and $G+C$ contents of $67 \cdot 2-71 \cdot 5 \mathrm{~mol} \%$. Furthermore, the polyphasic assignment of these strains to the genus Microbacterium was confirmed by the results of $16 \mathrm{~S}$ rDNA sequence comparisons and phylogenetic analyses which included all previously validly described Microbacterium species (Takeuchi \& Hatano, 1998).

This paper describes the characterization of these 11 strains. On the basis of morphological and chemotaxonomic characteristics, DNA-DNA hybridization data and previously obtained results of the $16 \mathrm{~S}$ rDNA sequence analysis (Takeuchi \& Hatano, 1998), we concluded that 10 out of the 11 strains represent six new Microbacterium species and one new combination in the genus Microbacterium, while the remaining strain was identified as Microbacterium laevaniformans.

\section{METHODS}

Bacterial strains and culture conditions. Strains used in this study are listed in Table 1. Microbacterium ketosireducens IFO $14548^{\mathrm{T}}$ and IFO 14549 , which reduce 2-5-diketoD-gluconic acid (2,7-anhydro- $\beta$-arabino-2-5-hepto-diulopyranose) to 2-keto-gulonic acid (2,7-anhydro- $\beta$-D-idoheptulopyranose), were isolated from soil in 1983, and 
Table 1. Bacterial strains used

Brackets indicate misidentifed name. ${ }^{\text {T }}$, Type strain. ATCC, American Type Culture Collection, Rockville, USA; BUCSAV, Biologicky Ustav. Czech Akademie Ved, Prague, Czech Republic; CDC, Centers for Disease Control and Prevention, Atlanta, USA; LCDC, Laboratory Center for Disease Control Tunney's Pasture, Ottawa, Canada; NCIB (= NCIMB, NCMB), National Collection of Industrial and Marine Bacteria, Aberdeen, UK; NCFB, National Collection of Food Bacteria, Reading Laboratory, Reading, UK.

\begin{tabular}{|c|c|c|c|c|}
\hline Proposed reclassification & Species & Strain (IFO no.) & Other designation & Source \\
\hline Microbacterium ketosireducens & 'Aureobacterium ketoreductum' & $14548^{\mathrm{T}}$ & & Soil \\
\hline Microbacterium ketosireducens & 'Aureobacterium ketoreductum' & 14549 & & Soil \\
\hline Microbacterium chocolatum & 'Chromobacterium chocolatum' & $3758^{\mathrm{T}}$ & BUCSAV $207^{\tau}$, NCIB $8181^{\mathrm{r}}$ & Culture contamination \\
\hline Microbacterium maritypicum & [Flavobacterium marinotypicum] & $15779^{\mathrm{T}}$ & ATCC $19260^{\mathrm{T}}, \mathrm{NCMB} 1050^{\mathrm{T}}$ & Sea water and marine mud \\
\hline Microbacterium aurantiacum & [Microbacterium laevaniformans] & $15234^{\mathrm{T}}$ & NCFB $2288^{\mathrm{T}}$, ATCC $49090^{\mathrm{T}}$ & Sewage \\
\hline Microbacterium aurantiacum & [Microbacterium laevaniformans] & 15235 & NCFB 2289, ATCC 49091 & Sewage \\
\hline Microbacterium hominis & Microbacterium sp. (CDC group A-4) & $15708^{\mathrm{T}}$ & LCDC $84-209^{\mathrm{T}}$ & Lung aspirate \\
\hline Microbacterium laevaniformans & Microbacterium sp. (CDC group A-5) & 15709 & LCDC $91-039^{T}$ & Blood \\
\hline Microbacterium thalassium & Strain No. 10 & $16060^{\mathrm{T}}$ & & Mangrove rhizosphere \\
\hline Microbacterium thalassium & Strain No. 71 & 16061 & & Mangrove rhizosphere \\
\hline Microbacterium halophilum & Strain No. 76 & $16062^{\mathrm{T}}$ & & Mangrove rhizosphere \\
\hline
\end{tabular}

maintained at the Institute for Fermentation, Osaka, as 'Aureobacterium ketoreductum'. Microbacterium chocolatum was isolated from culture contamination by $\mathrm{M}$. $\mathrm{H}$. Knutsen in 1944 (Clise, 1948). Microbacterium maritypicum appeared on the Approved List of Bacterial Names (Skerman et al., 1980) as [Flavobacterium marinotypicum] (Breed, 1957), but it is excluded from the genus Flavobacterium because it is Gram-positive and motile (Holmes et al., 1984). Microbacterium aurantiacum IFO $15234^{\mathrm{T}}$ and IFO 15235 were isolated from sewage and identified as Microbacterium laevaniformans. But they differ from the type strain of $M$. laevaniformans IFO $14471^{\mathrm{T}}$ in menaquinone composition and amino acid composition of the peptidoglycan. On the basis of the amino acids in their cell walls, the organisms should be excluded from the genus Microbacterium and probably be assigned to the genus Aureobacterium (Yokota et al., 1993b). Microbacterium hominis IFO $15708^{\mathrm{T}}$ and Microbacterium laevaniformans IFO 15709 were isolated from clinical specimens (Funke et al., 1995). Microbacterium thalassium IFO $16060^{\mathrm{T}}$ and IFO 16061, and Microbacterium halophilum IFO $16062^{\mathrm{T}}$ were isolated from soil in mangrove rhizospheres in Okinawa prefecture. For chemotaxonomic studies, each strain was cultured at $28^{\circ} \mathrm{C}$ with aerobic shaking in peptone-yeast extract medium (PY medium), which contained $1 \%$ peptone, $0.2 \%$ yeast extract, $0.2 \% \mathrm{NaCl}$ and $0.2 \% \mathrm{D}$-glucose $(\mathrm{pH} 7 \cdot 2)$, and then harvested by centrifugation during the stationary phase, washed with water and lyophilized.

Morphological, physiological and biochemical characterization. Cell morphology was determined by phase-contrast microscopy following cell growth on PY agar. Motility was determined by the hanging drop method. Unless otherwise indicated, biochemical tests were performed at $28^{\circ} \mathrm{C}$. Catalase activity was determined by the presence of bubbles in a $3 \%$ hydrogen peroxide solution. Acid production from carbohydrates was studied in a medium containing $1 \%$ peptone, $0.5 \% \mathrm{NaCl}, 0.003 \%$ bromothymol blue and $0.5 \%$ carbohydrate $(\mathrm{pH} 7 \cdot 2)$. Assimilation of carbohydrates and organic acids was studied in a medium containing $0.1 \%$ $\mathrm{NH}_{4} \mathrm{NO}_{3}, 0.1 \% \mathrm{~K}_{2} \mathrm{HPO}_{4}, 0.05 \% \quad \mathrm{MgSO}_{4} .7 \mathrm{H}_{2} \mathrm{O}, 0.02 \%$ $\mathrm{KCl}$ and $0.5 \%$ of carbohydrates or organic acids ( $\mathrm{pH} 7.0$ ). Nitrate reduction and hydrolysis of starch, aesculin, gelatin and Tweens 20, 40, 60 and 80 were tested by the methods described by Cowan (1974). API 20 NE tests were also used to determine physiological and biochemical characteristics.

Cell wall sugar analysis. Cell walls were prepared as described by Schleifer \& Kandler (1972). They were hydrolysed with $2 \mathrm{M} \mathrm{HCl}$ at $100{ }^{\circ} \mathrm{C}$ for $2 \mathrm{~h}$, dried in vacuo, then analysed as described by Mikami \& Ishida (1983), using a model LC-5A HPLC apparatus (Shimadzu) equipped with a Shim-pack ISA $07 / \mathrm{S} 2504$ column $(250 \times 4 \mathrm{~mm})$ and a Shimadzu model RF 530 spectrofluorometer.

Analysis of cellular fatty acids. Fatty acids were extracted from dried cells, purified, and analysed by GLC-MS with a GCMS-QP5000 spectrometer (Shimadzu) combined with a CLASS-5000 MS Workstation computer system as described previously (Takeuchi \& Hatano, 1998).

DNA-DNA hybridization. DNA-DNA hybridization was performed fluorometrically in microdilution wells by using biotinylated DNA (Ezaki et al., 1989).

\section{RESULTS AND DISCUSSION}

\section{Morphological, physiological and biochemical characteristics}

All 11 strains were Gram-positive, irregular short rods ranging from 0.2 to 0.5 by 0.6 to $2.0 \mu \mathrm{m}$. Many cells were arranged at angles, forming $\mathrm{V}$ shapes, but primary branching was not noted. In older cultures, rods were shorter, but a marked rod-coccus growth cycle did not occur. Colonies on solid media were circular, low convex with entire margins, opaque and moist. The pigments of the colonies of $M$. aurantiacum IFO $15234^{\mathrm{T}}$, IFO 15235 and $M$. chocolatum IFO $3758^{\mathrm{T}}$ were orange, and those of the other eight strains were light yellow, yellowish-white or yellow. M. maritypicum IFO $15779^{\mathrm{T}}$ was motile, but the other strains were non-motile. Two strains of $M$. thalassium, IFO $16060^{\mathrm{T}}$ and IFO 16061 , and two strains of $M$. ketosireducens, IFO $14548^{\mathrm{T}}$ and IFO 14549 , did not grow at $37^{\circ} \mathrm{C}$, but the other seven strains grew well. All strains except for M. chocolatum IFO $3758^{\mathrm{T}}$ grew 
Table 2. Phenotypic characteristics of Microbacterium species

Strains: $1, M$. ketosireducens IFO $14548^{\mathrm{T}}$, IFO $14549 ; 2, M$. chocolatum IFO $3758^{\mathrm{T}} ; 3, M$. maritypicum IFO $15779^{\mathrm{T}} ; 4, M$. aurantiacum IFO $15234^{\mathrm{T}}$, IFO 15235; $5, M$. hominis IFO $15708^{\mathrm{T}}$; $6, M$. thalassium IFO $16060^{\mathrm{T}}$, IFO $16061 ; 7, M$. halophilum IFO $16062^{\mathrm{T}} ; 8, M$. laevaniformans IFO15709; $9, M$. laevaniformans IFO14471 ${ }^{\mathrm{T}}$. Abbreviations: + , all strains positive; $+\mathrm{w}$, weakly positive; -, all strains negative; ND, not determined; Y, yellow; YW, yellowish white; LY, light yellow; $\mathrm{O}$, orange.

\begin{tabular}{|c|c|c|c|c|c|c|c|c|c|}
\hline Characteristic & $\mathbf{1}$ & 2 & 3 & 4 & 5 & 6 & 7 & 8 & 9 \\
\hline Colour of colonies & $\mathrm{Y}$ & $\mathrm{O}$ & LY & $\mathrm{O}$ & YW & $\mathrm{Y}$ & YW & YW & $\mathbf{Y}$ \\
\hline Motility & - & - & + & - & - & - & - & - & - \\
\hline Growth at $37^{\circ} \mathrm{C}$ & - & + & + & + & + & - & + & + & + \\
\hline Growth in $2 \% \mathrm{NaCl}$ & + & $+w$ & + & + & + & + & + & + & + \\
\hline Growth in $5 \% \mathrm{NaCl}$ & $+w$ & - & + & + & $+\mathrm{w}$ & + & + & + & ND \\
\hline Growth in $6.5 \% \mathrm{NaCl}$ & - & - & $+w$ & $+\mathrm{w}$ & $+\mathrm{w}$ & $+w$ & + & - & ND \\
\hline \multicolumn{10}{|l|}{ Hydrolysis of: } \\
\hline Gelatin & + & - & + & - & - & $+w /-$ & $+w$ & - & - \\
\hline Starch & + & $+w$ & - & + & - & + & + & + & + \\
\hline $\mathrm{H}_{2} \mathrm{~S}$ formation & + & + & - & + & + & - & - & + & + \\
\hline MR test & + & - & - & - & + & + & + & + & + \\
\hline VP test & - & - & - & - & + & - & - & + & + \\
\hline \multicolumn{10}{|l|}{ Assimilation of: } \\
\hline Arabinose & + & - & - & $-/+w$ & + & - & - & - & - \\
\hline Maltose & + & + & + & + & + & + & + & + & + \\
\hline Mannose & + & $+w$ & + & + & + & + & + & + & + \\
\hline Mannitol & + & $+w$ & + & + & + & + & + & + & + \\
\hline$N$-Acetylglucosamine & - & - & + & - & + & - & - & - & - \\
\hline Acetate & $+w$ & - & - & - & + & - & - & + & + \\
\hline Adipate & - & - & - & - & - & - & - & - & - \\
\hline Caprate & - & - & - & - & - & - & - & - & - \\
\hline Citrate & $+1-$ & - & + & - & + & - & - & - & + \\
\hline Fumarate & $+w$ & - & - & + & + & - & - & + & + \\
\hline Gluconate & + & + & + & + & + & + & + & + & + \\
\hline Lactate & $+\mathrm{w}$ & - & - & $-/+w$ & + & - & - & + & + \\
\hline Malate & - & - & + & $-/+w$ & + & - & - & + & + \\
\hline Phenyl acetate & - & - & + & - & - & - & - & - & - \\
\hline Propionate & - & - & - & + & + & - & - & + & + \\
\hline \multicolumn{10}{|l|}{ Acid produced from: } \\
\hline L-Arabinose & + & - & - & - & + & - & - & - & - \\
\hline Galactose & $+1-$ & - & - & - & + & - & - & + & + \\
\hline Glucose & + & - & + & $+w /-$ & + & + & + & + & + \\
\hline Inulin & $-/+w$ & - & - & - & - & - & - & - & - \\
\hline Mannose & + & + & + & + & + & + & + & + & + \\
\hline Melezitose & - & - & - & - & + & - & - & - & - \\
\hline Raffinose & - & - & - & - & - & - & - & - & + \\
\hline Rhamnose & - & - & - & - & - & - & + & - & + \\
\hline Ribose & - & - & - & - & - & - & - & - & $\mathrm{ND}$ \\
\hline Sucrose & + & + & $+w$ & + & + & - & - & - & + \\
\hline Trehalose & - & - & - & - & + & - & - & - & + \\
\hline Xylose & + & + & - & $-/+$ & - & + & - & - & - \\
\hline
\end{tabular}

weakly in broth media containing $5 \% \mathrm{NaCl} . M$. halophilum IFO $16062^{\mathrm{T}}$ grew well in broth media containing $6.5 \% \mathrm{NaCl}$, but other strains grew weakly or not at all. The other physiological properties of the 11 strains and $M$. laevaniformans IFO $14471^{\mathrm{T}}$, which was added as a reference strain, are shown in Table 2.
Two strains of $M$. aurantiacum IFO $15234^{\mathrm{T}}$ and IFO 15235 , two strains of $M$. ketosireducens IFO $14548^{\mathrm{T}}$ and IFO 14549, and two strains of $M$. thalassium IFO $16060^{\mathrm{T}}$ and IFO 16061, showed identical phenotypic characteristics, respectively, and Microbacterium sp. IFO 15709 was similar to $M$. laevaniformans IFO 
Table 3. Cellular fatty acids of Microbacterium species

\begin{tabular}{|c|c|c|c|c|c|c|c|c|c|}
\hline \multirow[t]{2}{*}{ Species } & \multirow{2}{*}{$\begin{array}{c}\text { Strain } \\
\text { (IFO no.) }\end{array}$} & \multicolumn{8}{|c|}{ Cellular fatty acid composition (\%) } \\
\hline & & i14:0 & i15:0 & ai15:0 & i16:0 & $16: 0$ & i17:0 & ai17:0 & 18:0 \\
\hline M. ketosireducens & $14548^{\mathrm{T}}$ & - & $11 \cdot 3$ & $34 \cdot 9$ & $19 \cdot 0$ & $3 \cdot 2$ & $6 \cdot 7$ & $22 \cdot 0$ & $2 \cdot 9$ \\
\hline M. ketosireducens & 14549 & - & $11 \cdot 9$ & $34 \cdot 4$ & $18 \cdot 1$ & $3 \cdot 6$ & $6 \cdot 8$ & $20 \cdot 4$ & $4 \cdot 8$ \\
\hline M. chocolatum & $3758^{\mathrm{T}}$ & - & $6 \cdot 3$ & $34 \cdot 7$ & $10 \cdot 9$ & $4 \cdot 6$ & $4 \cdot 7$ & $38 \cdot 8$ & - \\
\hline M. maritypicum & $15779^{\mathrm{T}}$ & $1 \cdot 3$ & $5 \cdot 8$ & $63 \cdot 6$ & $21 \cdot 3$ & $1 \cdot 3$ & - & $6 \cdot 7$ & - \\
\hline M. aurantiacum & $15234^{\mathrm{T}}$ & - & $13 \cdot 1$ & $34 \cdot 2$ & $12 \cdot 2$ & $5 \cdot 8$ & $7 \cdot 9$ & $24 \cdot 3$ & $2 \cdot 5$ \\
\hline M. aurantiacum & 15235 & - & $13 \cdot 0$ & $30 \cdot 0$ & $12 \cdot 9$ & $8 \cdot 0$ & $7 \cdot 8$ & $21 \cdot 7$ & $6 \cdot 6$ \\
\hline M. hominis & $15708^{\mathrm{T}}$ & - & $5 \cdot 7$ & $40 \cdot 9$ & $21 \cdot 0$ & $6 \cdot 7$ & $2 \cdot 3$ & $23 \cdot 5$ & - \\
\hline M. laevaniformans & 15709 & - & $4 \cdot 0$ & $36 \cdot 1$ & $20 \cdot 6$ & $7 \cdot 5$ & - & $26 \cdot 8$ & $5 \cdot 0$ \\
\hline M. thalassium & $16060^{\mathrm{T}}$ & - & $10 \cdot 1$ & $30 \cdot 5$ & $25 \cdot 7$ & $2 \cdot 6$ & $6 \cdot 4$ & $24 \cdot 7$ & - \\
\hline M. thalassium & 16061 & - & $21 \cdot 4$ & $25 \cdot 8$ & $28 \cdot 0$ & $2 \cdot 6$ & $9 \cdot 2$ & $13 \cdot 0$ & - \\
\hline M. halophilum & $16062^{\mathrm{T}}$ & 0.9 & $22 \cdot 3$ & $24 \cdot 0$ & $23 \cdot 5$ & $3 \cdot 9$ & $11 \cdot 3$ & $12 \cdot 1$ & $2 \cdot 0$ \\
\hline
\end{tabular}

Table 4. Chemotaxonomic characteristics of Microbacterium species

Data from this study and previous studies (Takeuchi \& Hatano, 1998). Parentheses indicate that a trace amount is present. Abbreviations: ai, anteiso-branched acid; i, iso-branched acid; Hsr, homoserine; Orn, ornithine; Lys, lysine; Rha, rhamnose; Gal, galactose; Glc, glucose; 6dT, 6-deoxytalose; Man, mannose; Fuc, fucose; Xyl, xylose.

\begin{tabular}{|c|c|c|c|c|c|c|c|}
\hline \multirow[t]{3}{*}{ Species } & \multirow{3}{*}{$\begin{array}{c}\text { Strain } \\
\text { (IFO no.) }\end{array}$} & \multirow{3}{*}{$\begin{array}{c}G+C \text { content } \\
(\mathrm{mol} \%)\end{array}$} & \multirow{3}{*}{$\begin{array}{c}\text { Menaquinone } \\
\text { (MK) }\end{array}$} & \multirow[t]{3}{*}{ Cellular fatty acid } & \multicolumn{3}{|c|}{ Cell wall composition } \\
\hline & & & & & \multicolumn{2}{|c|}{ Amino acid } & \multirow[t]{2}{*}{ Sugar } \\
\hline & & & & & Position 3 & Linkage & \\
\hline M. ketosireducens & $14548^{\mathrm{T}}$ & $69 \cdot 8$ & 13 & ai15, ai17, i16 & $\mathrm{Hsr}$ & Orn & $\mathrm{ND}$ \\
\hline M. ketosireducens & 14549 & $69 \cdot 7$ & 13 & ail 5 , ai17, i16 & $\mathrm{Hsr}$ & Orn & ND \\
\hline M. chocolatum & $3758^{\mathrm{T}}$ & $69 \cdot 5$ & 12 & ai 15 , ai 17, i 16 & $\mathrm{Hsr}$ & Orn & $\begin{array}{l}\text { Rha, Gal, (Man), } \\
\text { (Xyl) }\end{array}$ \\
\hline M. maritypicum & $15779^{\mathrm{T}}$ & $71 \cdot 6$ & 12 & ai15, (ai17), i16 & Hsr & Orn & $\mathrm{Gal}$ \\
\hline M. aurantiacum & $15234^{\mathrm{T}}$ & $70 \cdot 3$ & 12 & ai15, ai17, i16 & $\mathrm{Hsr}$ & Orn & Rha, Gal, Fuc \\
\hline M. aurantiacum & 15235 & $70 \cdot 1$ & 12 & ai 15, ail 7, i16 & $\mathrm{Hsr}$ & Orn & Rha, Gal, Fuc \\
\hline M. hominis & $15708^{\mathrm{T}}$ & $71 \cdot 2$ & 11,12 & ai 15, ai1 7, i16 & Lys & Lys & Rha, 6dT, Gal, Man \\
\hline M. laevaniformans & 15709 & $68 \cdot 8$ & 11,12 & ai 15, ai 17, il 6 & Lys & Lys & $\begin{array}{l}\text { Rha, Gal, (Glc), } \\
\text { (Man) }\end{array}$ \\
\hline M. thalassium & $16060^{\mathrm{T}}$ & $69 \cdot 7$ & 11,12 & ai15, ai17, i16 & $\mathrm{Hsr}$ & Orn & Gal, Glc \\
\hline M. thalassium & 16061 & $69 \cdot 1$ & 11,12 & ai 15, ail 7, i1 5, i16 & $\mathrm{Hsr}$ & Orn & Rha, Man, Gal, Glc \\
\hline M. halophilum & $16062^{\mathrm{T}}$ & $67 \cdot 2$ & $11,12,13$ & ai 15, ail 7, i1 5, il 6 & $\mathrm{Hsr}$ & Orn & Man, Gal, Glc \\
\hline
\end{tabular}

$14474^{\mathrm{T}}$, but differed in such features as assimilation of citrate and acid production from some carbohydrates.

\section{Chemical characteristics}

As shown in Table 3, cellular fatty acids of all strains except $M$. maritypicum IFO $15779^{\mathrm{T}}$ and two strains isolated from mangrove rhizosphere, $M$. thalassium IFO 16061 and $M$. halophilum IFO $16062^{\mathrm{T}}$, were composed mainly of anteiso-C15:0, anteiso-C17:0 and iso-C16:0. In $M$. maritypicum IFO $15779^{\mathrm{T}}$, anteiso-C17:0 was detected as a minor component. In $M$. thalassium IFO 16061 and $M$. halophilum IFO $16062^{\mathrm{T}}$, iso-C15:0 was detected as a major component. Chemotaxonomic characteristics of the 11 strains are summarized in Table 4. The cell wall sugars rhamnose, galactose, fucose, mannose, 6-deoxytalose, mannose and glucose were detected in various combinations.

\section{DNA-DNA hybridization}

High DNA-DNA relatedness values $(>70 \%)$ were obtained between two strains of $M$. aurantiacum IFO $15234^{\mathrm{T}}$ and IFO 15235 , two strains of $M$. ketosireducens IFO $14548^{\mathrm{T}}$ and IFO 14549 , two strains of $M$. thalassium IFO $16060^{\mathrm{T}}$ and IFO 16061, and Microbacterium sp. IFO 15709 and M. laevaniformans IFO $14471^{\mathrm{T}}$ (Table 5), but none of these pairs showed high homology values with each other or with previously described species of the genus Microbacterium. These results indicate that each of these strains constitutes a separate taxon.

The conclusions described above are supported by phylogenetic analysis (Takeuchi \& Hatano, 1998). We previously determined almost complete 16S rDNA sequences of these 11 strains, and compared them with published sequences of species included in the genus 
Table 5. Levels of DNA-DNA similarity among strains of the genus Microbacterium

\begin{tabular}{|c|c|c|c|c|c|c|c|c|c|c|c|c|c|}
\hline Species & $\begin{array}{c}\text { Strain } \\
\text { (IFO No.) }\end{array}$ & $15234^{\mathrm{T}}$ & 15235 & $3758^{\mathrm{T}}$ & $16062^{\mathrm{T}}$ & $14548^{\mathrm{T}}$ & 14549 & $14471^{T}$ & 15709 & $15779^{\mathrm{T}}$ & $15708^{\mathrm{T}}$ & $16060^{\mathrm{T}}$ & 16061 \\
\hline M. arabinogalactanolyticum & $14344^{T}$ & 11 & 2 & 17 & 19 & 12 & 13 & 15 & ND & 10 & 19 & 10 & 11 \\
\hline M. arborescens & $3750^{\mathrm{T}}$ & 17 & $\mathrm{ND}$ & 6 & 15 & 36 & ND & 10 & 4 & 17 & 11 & 14 & 10 \\
\hline M. aurantiacum & $15234^{\mathrm{T}}$ & 100 & 83 & 38 & ND & 29 & 16 & 18 & ND & 11 & 13 & ND & ND \\
\hline M. aurantiacum & 15235 & 86 & 100 & 44 & ND & 23 & 20 & 23 & ND & 13 & 23 & ND & ND \\
\hline M. aurum & $15204^{\mathrm{T}}$ & 38 & ND & 10 & 8 & 36 & ND & 19 & 13 & 7 & 16 & 7 & 17 \\
\hline M. barkeri & $15036^{\mathrm{T}}$ & 5 & 1 & 4 & 36 & 11 & 9 & 10 & ND & 15 & 3 & 15 & 8 \\
\hline$M$. chocolatum & $3758^{\mathrm{T}}$ & 51 & 46 & 100 & ND & 30 & 21 & 13 & ND & 21 & 19 & ND & ND \\
\hline M. dextranolyticum & $14592^{\mathrm{T}}$ & 16 & $\mathrm{ND}$ & 11 & 11 & 10 & $\mathrm{ND}$ & 10 & 14 & 14 & 13 & 12 & 9 \\
\hline M. esteraromaticum & $3751^{\mathrm{T}}$ & 6 & 1 & 6 & 37 & 12 & 10 & 12 & ND & 11 & 13 & 7 & 11 \\
\hline$M$. flavescens & $15039^{\mathrm{T}}$ & 29 & 10 & 23 & 31 & 30 & 56 & 7 & $\mathrm{ND}$ & 13 & 18 & 16 & 12 \\
\hline M. halophilum & $16062^{\mathrm{T}}$ & ND & ND & ND & 100 & ND & ND & ND & ND & $\mathrm{ND}$ & ND & 21 & 15 \\
\hline M. imperiale & $12610^{\mathrm{T}}$ & 28 & ND & 11 & 14 & 13 & ND & 14 & 13 & 6 & 25 & 7 & 21 \\
\hline M. keratanolyticum & $13309^{\mathrm{T}}$ & 10 & 2 & 7 & 49 & 11 & 17 & 10 & ND & 9 & 34 & 9 & 7 \\
\hline M. ketosireducens & $14548^{\mathrm{T}}$ & 16 & 12 & 18 & ND & 100 & 95 & 13 & ND & 9 & 15 & ND & ND \\
\hline M. ketosireducens & 14549 & 15 & 8 & 13 & 35 & 76 & 100 & 19 & ND & 11 & 25 & ND & ND \\
\hline M. lacticum & $14135^{T}$ & 19 & ND & 15 & 31 & 35 & ND & 14 & 37 & 28 & 25 & 16 & 7 \\
\hline M. laevaniformans & $14471^{\mathrm{T}}$ & 17 & ND & 16 & 16 & 31 & ND & 100 & 90 & 17 & 25 & 17 & 12 \\
\hline M. laevaniformans & 15709 & 23 & $\mathrm{ND}$ & 23 & ND & 26 & ND & 81 & 100 & ND & 17 & ND & ND \\
\hline$M$. liquefaciens & $15037^{\mathrm{T}}$ & 6 & 3 & 5 & 14 & 11 & 14 & 5 & ND & 13 & 6 & 12 & 10 \\
\hline M. luteolum & $15074^{\mathrm{T}}$ & 25 & 13 & 36 & 11 & 40 & 36 & 20 & ND & 27 & 10 & 9 & 7 \\
\hline M. maritypicum & $15779^{\mathrm{T}}$ & 9 & 1 & 20 & ND & 9 & 8 & 22 & ND & 100 & 39 & ND & ND \\
\hline M. hominis & $15708^{\mathrm{T}}$ & 21 & ND & 11 & $\mathrm{ND}$ & 21 & ND & 18 & 7 & 39 & 100 & ND & ND \\
\hline M. saperdae & $15038^{\mathrm{T}}$ & 10 & 7 & 6 & 13 & 14 & 23 & 18 & ND & 19 & 7 & 12 & 8 \\
\hline M. schleiferi & $15075^{\mathrm{T}}$ & 20 & 13 & 14 & 21 & 21 & 21 & 19 & ND & 9 & 12 & 14 & 7 \\
\hline M. terrae & $15300^{\mathrm{T}}$ & 4 & 8 & 5 & 13 & 47 & 41 & 28 & ND & 10 & 30 & 15 & 8 \\
\hline M. terregens & $12961^{T}$ & 9 & 7 & 11 & 6 & 31 & 32 & 25 & $\mathrm{ND}$ & 13 & 9 & 17 & 7 \\
\hline M. testaceum & $12675^{\mathrm{T}}$ & 20 & 2 & 9 & 41 & 15 & 9 & 23 & ND & 15 & 14 & 14 & 9 \\
\hline M. thalassium & $16060^{T}$ & ND & ND & ND & 42 & $\mathrm{ND}$ & ND & ND & ND & ND & ND & 100 & 76 \\
\hline M. thalassium & 16061 & ND & ND & ND & 41 & ND & ND & ND & $\mathrm{ND}$ & ND & ND & 79 & 100 \\
\hline M. trichothecenolyticum & $15077^{\mathrm{T}}$ & 9 & 5 & 13 & 24 & 15 & 18 & 5 & ND & 13 & 7 & 13 & 12 \\
\hline
\end{tabular}

ND, Not determined.

Microbacterium and other members of the family Microbacteriaceae (Takeuchi \& Hatano, 1998). As shown in Fig. 1, the 16S rDNA sequences of these strains represent seven distinct lines of descent within the genus Microbacterium. These correspond to $M$. maritypicum IFO $15779^{\mathrm{T}}, M$. ketosireducens IFO $14548^{\mathrm{T}}, M$. thalassium IFO $16060^{\mathrm{T}}, M$. aurantiacum IFO $15234^{\mathrm{T}}, M$. chocolatum IFO $3758^{\mathrm{T}}, M$. hominis IFO $15708^{\mathrm{T}}$ and $M$. halophilum IFO $16062^{\mathrm{T}}$, respectively.

On the basis of morphological, physiological and chemotaxonomic characteristics, together with DNA-DNA hybridization and 16S rDNA sequence comparison results, we conclude that these organisms should be classified as new species in the genus Microbacterium, and propose six new species and one new combination in the genus Microbacterium: Microbacterium ketosireducens sp. nov. (type strain IFO $14548^{\mathrm{T}}$ ), Microbacterium chocolatum sp. nov. (type strain IFO $3758^{\mathrm{T}}$ ), Microbacterium aurantiacum $\mathrm{sp}$. nov. (type strain IFO 15234 ), Microbacterium hominis sp. nov. (type strain IFO $15708^{\mathrm{T}}$ ), Microbacterium thalassium sp. nov. (type strain IFO $16060^{\mathrm{T}}$ ), Microbacterium halophilum sp. nov. (type strain IFO $16062^{\mathrm{T}}$ ) and Microbacterium maritypicum comb. nov. (type strain IFO $15779^{\mathrm{T}}$ ).

Nineteen species have hitherto been accommodated in the genus Microbacterium. From the results of this study, seven species have been added to the genus
Microbacterium, and 26 species are accommodated in this genus. Differential characteristics for these species and the 19 previously described species of the genus Microbacterium are summarized in Table 6. These results show that species in the genus Microbacterium can be separated from each other by their morphological, physiological and chemotaxonomic characteristics.

Microbacterium species were widely distributed in various environments such as soil [Microbacterium arabinogalactanolyticum, Microbacterium keratanolyticum, Microbacterium luteolum, Microbacterium schleiferi, Microbacterium terrae, Microbacterium trichothecenolyticum (Yokota et al., 1993a), Microbacterium flavescens, Microbacterium terregens, Microbacterium testaceum (Collins et al., 1983) and Microbacterium dextranolyticum (Yokota et al., 1993b)], sewage [Microbacterium barkeri (Collins et al., 1983) and Microbacterium laevaniformans (Collins et al., 1986)], lake water [Microbacterium arborescens (Breed, 1957)], steep liquor [Microbacterium aurum (Yokota et al., 1993b)], milk products or cheese [Microbacterium lacticum (Collins et al., 1986) and Microbacterium liquefaciens (Collins et al., 1983)]. In addition to these organisms, some Microbacterium species have been isolated from different sources; Microbacterium esteraromaticum was isolated from accidental contamination in culture (Breed, 1957; Yokota et al., 1993a). Microbacterium imperiale was isolated from the alimentary tract of the imperial moth Eacles imperialis 


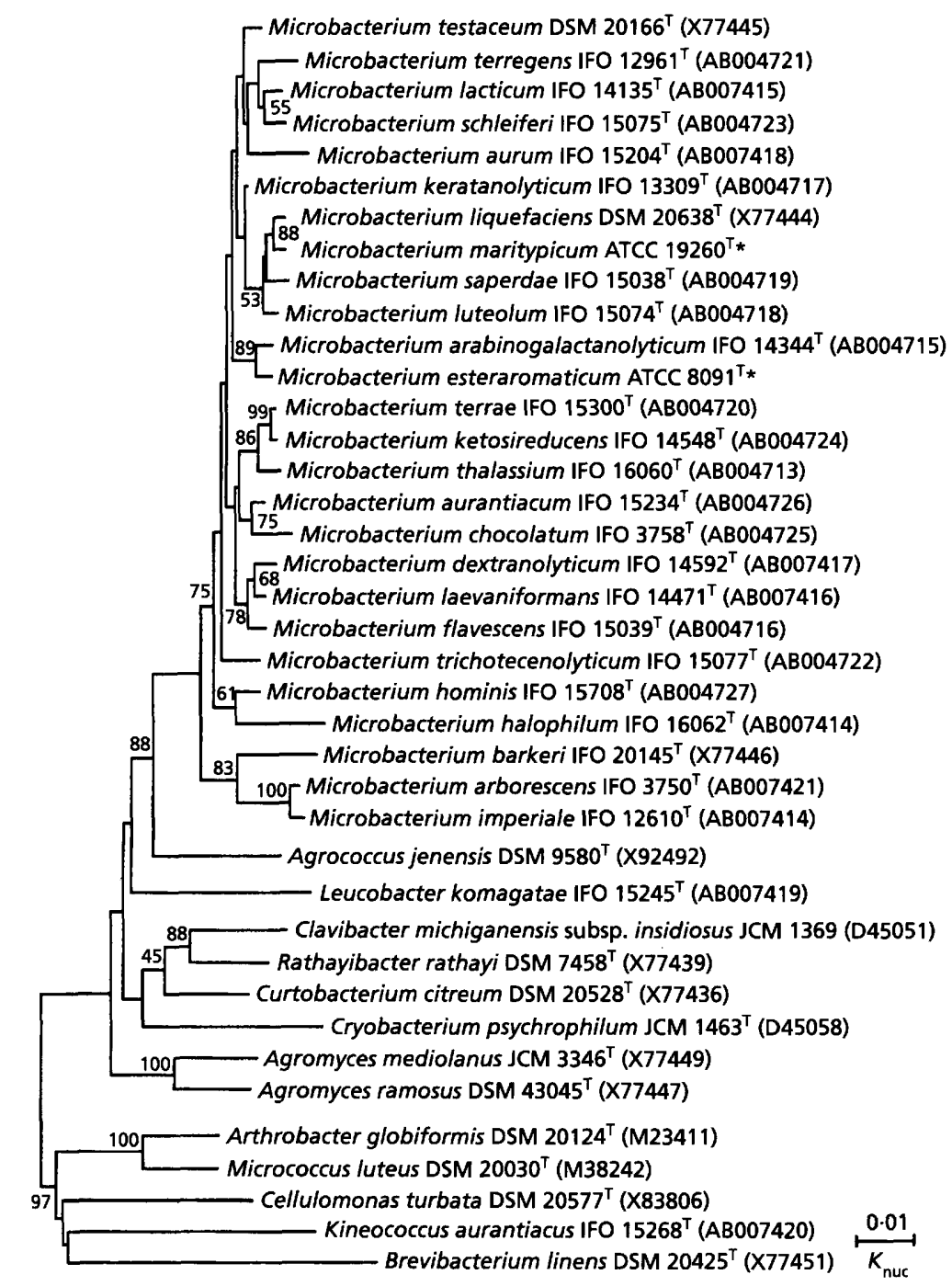

Fig. 1. Phylogenetic dendrogram based on a comparison of the 16S rDNA sequences of Microbacterium species and some close relatives (Takeuchi \& Hatano, 1998). The tree was created by using the neighbour-joining method and $K_{\text {nuc }}$ values. The numbers on the tree indicate the percentages of bootstrap samplings, derived from 1000 samples, supporting the internal branches (Felsenstein, 1985). Asterisks indicate that sequence data were obtained from the Ribosomal Database Project (RDP) (Larsen et al., 1993).
Dru (Collins et al., 1986), and Microbacterium saperdae was isolated from dead larvae (Collins et al., 1983). Recently, Microbacterium spp. and other actinobacteria were isolated from the surface of cheese (Kollöffel et al., 1997), and Aureobacterium spp. were isolated from clinical specimens (Saweljew et al., 1996).

As shown in Table 1, each species which was identified as a new species or a new combination in the genus Microbacterium, was isolated from soil (M. ketosireducens, $M$. halophilum and $M$. thalassium), culture contamination ( $M$. chocolatum), sea water or marine mud $[M$. maritypicum (Breed, 1957)], sewage ( $M$. aurantiacum) or clinical specimens ( $M$. hominis and $M$. laevaniformans). Among the species isolated from soil, $M$. thalassium and $M$. halophilum were isolated from soil in mangrove rhizosphere in Okinawa prefecture, Japan, where complex environments have formed under the influence of tidal ebb and flow, the influx of fresh water, and high temperature and humidity in these subtropical and tropical climates. The soils in such environments are muddy, and are reported to be anoxic, low in nutrients, and to have higher concentrations of heavy metals and higher salinity than terrestrial soils (Wakushima et al., 1994). These results also show the variety of environments in which Microbacterium species are distributed.

\section{Description of Microbacterium aurantiacum sp. nov.}

Microbacterium aurantiacum (au.ran.ti'a.cum. L. n. aurantium specific name of the orange; M.L. neut. adj. aurantiacum orange-coloured).

Good growth occurs on solid media in air; colonies are $2-4 \mathrm{~mm}$ in diameter, circular, low convex with entire margins, opaque and moist. Orange pigment is produced. In young cultures, small, slender rods are formed. Many cells are arranged at angles, forming V shapes; primary branching is uncommon. In older cultures, rods are shorter, but a marked rod-coccus growth cycle does not occur. Gram-positive nonmotile rods. The optimum growth temperature is about $28^{\circ} \mathrm{C}$, and growth occurs at $37^{\circ} \mathrm{C}$. Growth 
Table 6. Differential characteristics of Microbacterium species

Abbreviations: + , all strains positive; + w, weakly positive; - , all strains negative; d, differs among strains; ND, not determined;Y, yellow; YW, yellowish white; LY, light yellow; O, orange; GEL, gelatin; STA, starch; VP test, Voges-Proskauer test; ADH, arginine dihydrolase; ARA, arabinose; NAc-GlcN, $N$-acetylglucosamine; MLT, malate; CIT, citrate; PAC, phenyl acetate; Glc, glucose.

\begin{tabular}{|c|c|c|c|c|c|c|c|c|c|c|c|c|c|c|c|c|c|}
\hline \multirow[t]{2}{*}{ Species } & \multirow{2}{*}{$\begin{array}{l}\text { Colour of } \\
\text { colony }\end{array}$} & \multirow[t]{2}{*}{ Motility } & \multicolumn{2}{|c|}{ Growth } & \multicolumn{2}{|c|}{ Hydrolysis of: } & \multirow{2}{*}{$\begin{array}{c}\mathbf{H}_{2} \mathbf{S} \\
\text { prodn }\end{array}$} & \multirow{2}{*}{$\begin{array}{l}\text { VP } \\
\text { test }\end{array}$} & \multirow[t]{2}{*}{ ADH } & \multicolumn{5}{|c|}{ Assimilation of: } & \multirow{2}{*}{$\begin{array}{l}\text { Acid } \\
\text { from } \\
\text { Glc }\end{array}$} & \multirow{2}{*}{$\begin{array}{c}\text { Cell } \\
\text { wall } \\
\text { diamino }\end{array}$} & \multirow{2}{*}{$\begin{array}{c}\text { Major } \\
\text { menaquinone } \\
\text { acid }\end{array}$} \\
\hline & & & $37^{\circ} \mathrm{C}$ & $2 \% \mathrm{NaCl}$ & GEL & STA & & & & ARA & $\begin{array}{l}\text { NAc- } \\
\text { GlcN }\end{array}$ & MLT & CIT & PAC & & & \\
\hline M. arabinogalactanolyticum & YW & - & - & + & + & + & + & - & + & + & + & + & - & + & - & Orn & MK-12,13 \\
\hline M. arborescens & 0 & + & - & ND & + & - & + & - & - & + & + & + & $+w$ & - & + & Lys & MK-11,12 \\
\hline M. aurantiacum & $\mathrm{O}$ & - & + & + & - & + & + & - & - & $\mathrm{d}$ & - & $\mathrm{d}$ & - & - & $\mathrm{d}$ & Orn & MK-12 \\
\hline M. aurum & YW & - & + & ND & + & + & + & - & - & - & $+w$ & - & - & - & + & Lys & MK-11, 12 \\
\hline M. barkeri & W & + & + & + & + & + & + & - & + & + & + & + & + & - & - & Orn & $\mathrm{MK}-11,12$ \\
\hline$M$. chocolatum & $\mathrm{O}$ & - & + & $+w$ & - & $+w$ & + & - & - & - & $+w$ & - & - & - & - & Orn & $\mathrm{MK}-12$ \\
\hline M. dextranolyticum & w & - & - & $\mathrm{ND}$ & - & - & + & + & - & + & - & + & - & - & + & Orn & MK-11, 12 \\
\hline M. esteraromaticum & YW & + & $\mathrm{d}$ & - & - & + & + & - & - & + & - & - & - & - & - & Orn & MK-12, 13 \\
\hline M. flavescens & $\mathrm{Y}$ & - & - & + & + & + & + & - & - & + & - & + & - & - & + & Orn & MK-13, 14 \\
\hline M. halophilum & $\mathrm{Y}$ & - & + & + & $+w$ & + & - & - & - & - & - & - & - & - & + & Orn & MK-11, 12, 13 \\
\hline$M$. hominis & YW & - & + & + & - & - & + & + & - & + & + & + & + & - & + & Lys & MK- 11,12 \\
\hline M. imperiale & $\mathrm{O}$ & + & + & ND & - & + & + & - & - & + & $+w$ & + & - & - & + & Lys & MK-11, 12 \\
\hline M. keratanolyticum & $\mathrm{Y}$ & + & - & + & + & - & + & - & $+w$ & $+w$ & + & - & - & - & - & Orn & MK-12, 13 \\
\hline M. ketosireducens & $\mathrm{Y}$ & - & - & + & + & + & + & - & - & + & - & - & d & - & + & Orn & MK-13 \\
\hline M. lacticum & $\mathrm{Y}$ & - & - & ND & - & + & - & - & - & $+w$ & + & + & - & - & + & Lys & MK-11, 12 \\
\hline M. laevaniformans & $\mathrm{Y} / \mathrm{YW}$ & - & + & ND & $\mathrm{d}$ & + & + & + & $\mathrm{d}$ & - & - & + & - & - & + & Lys & MK-11, 12 \\
\hline M. liquefaciens & $\mathrm{Y}$ & - & - & + & + & - & + & - & + & - & + & - & + & - & - & Orn & MK-11, 12 \\
\hline M. luteolum & YW & - & - & - & - & - & + & - & - & + & + & + & - & - & - & Orn & MK-12 \\
\hline M. maritypicum & $\mathrm{Y}$ & + & + & + & + & - & - & - & - & - & + & + & + & + & + & Orn & MK-12 \\
\hline M. saperdae & YW & + & - & - & - & + & + & - & + & + & + & + & - & - & + & Orn & MK-11, 12 \\
\hline M. schleiferi & YW & - & $+w$ & $+w$ & - & - & - & + & - & $+w$ & $+w$ & + & - & - & + & Orn & MK-11, 12 \\
\hline M. terrae & $\mathrm{Y}$ & - & - & + & + & + & + & - & - & + & - & - & - & - & + & Orn & MK-13, 14 \\
\hline M. terregens & $\mathrm{Y}$ & - & - & $+w$ & - & - & - & - & - & - & - & + & - & - & + & Orn & MK-12, 13 \\
\hline$M$, testaceum & $\mathrm{O}$ & + & - & - & + & - & + & - & - & + & + & + & + & $+w$ & - & Orn & MK-10, 11 \\
\hline$M$. thalassium & Y/LY & - & - & + & $d$ & + & - & - & - & - & - & - & - & - & - & Orn & MK-11, 12 \\
\hline M. trichothecenolyticum & $\mathrm{Y}$ & - & - & $+w$ & - & + & + & - & - & - & + & + & + & - & + & Orn & $\mathrm{MK}-12,13$ \\
\hline
\end{tabular}

occurs in the presence of 2 and $5 \% \mathrm{NaCl}$, but is very weak in the presence of $6.5 \% \mathrm{NaCl}$. Maltose, mannose, mannitol, fumarate, gluconate and propionate are assimilated, but arabinose, $\mathrm{N}$-acetylglucosamine, acetate, adipate, caprate, citrate, lactate, malate and phenyl acetate are assimilated weakly or are not assimilated. Starch, Tweens 20, 40, 60 and 80 are hydrolysed. Gelatin is not hydrolysed. $\mathrm{H}_{2} \mathrm{~S}$ is produced. Methyl red test and Voges-Proskauer test are negative. Arginine dihydrolase is not produced. Acid is produced from glucose, mannose and sucrose, but is not produced or is produced weakly from L-arabinose, galactose, glucose, inulin, melezitose, raffinose, rhamnose, ribose, trehalose and xylose. The cell wall peptidoglycan contains ornithine, homoserine, glutamate plus hydroxyglutamate, glycine and alanine. The cell wall sugars are rhamnose, galactose and fucose. The major cellular fatty acids are anteiso$\mathrm{C} 15: 0$, anteiso-C17:0 and iso-C16:0. Unsaturated menaquinones with 12 isoprene units are present. The DNA $\mathrm{G}+\mathrm{C}$ content of the type strain is $70 \cdot 1-70 \cdot 3 \mathrm{~mol} \%$. Source is sewage. The type strain is IFO $15234^{\mathrm{T}}$.

\section{Description of Microbacterium chocolatum sp. nov.}

Microbacterium chocolatum (choc.o.lat.um. M.L. neut. adj. chocolatum chocolate-coloured, derived from the Mexican chocolatl, chocolate).
Good growth occurs on solid media in air; colonies are 2-4 mm in diameter, circular, low convex with entire margins, opaque and moist. Orange or dull orange pigment is produced. In young cultures, small, slender rods are formed. Many cells are arranged at angles, forming $\mathrm{V}$ shapes; primary branching is uncommon. In older cultures, rods are shorter, but a marked rod-coccus growth cycle does not occur. Grampositive non-motile rods. The optimum temperature for growth is about $28^{\circ} \mathrm{C}$, and growth occurs at $37^{\circ} \mathrm{C}$. Growth is weak in the presence of $2 \% \mathrm{NaCl}$, but does not occur in the presence of 5 and $6.5 \% \mathrm{NaCl}$. Maltose and gluconate are assimilated, but arabinose, mannose, mannitol, $\mathrm{N}$-acetylglucosamine, acetate, adipate, caprate, citrate, fumarate, lactate, malate, phenyl acetate and propionate are assimilated weakly or are not assimilated. Starch, Tweens 20, 40, 60 and 80 are hydrolysed. Gelatin is not hydrolysed. $\mathrm{H}_{2} \mathrm{~S}$ is produced. Methyl red test and Voges-Proskauer test are negative. Arginine dihydrolase is not produced. Acid is produced from mannose, sucrose and xylose, but is not produced from L-arabinose, galactose, glucose, inulin, melezitose, raffinose, rhamnose, ribose and trehalose. The cell wall peptidoglycan contains ornithine, homoserine, glutamate plus hydroxyglutamate, glycine and alanine. The cell wall sugars are rhamnose and galactose as major components, and mannose and xylose as minor components. The major cellular fatty acids are anteiso-C15:0, anteiso-C17:0 
and iso-C16:0. Unsaturated menaquinones with 12 isoprene units are present. The DNA G $+\mathrm{C}$ content of the type strain is $69.5 \mathrm{~mol} \%$. Source is culture contamination. The type strain is IFO $3758^{\mathrm{T}}$.

\section{Description of Microbacterium halophilum sp. nov.}

Microbacterium halophilum (ha.lo'phi.lum. Gr. n. hals salt; Gr. adj. philos loving; M.L. neut. adj. halophilum salt-loving).

Good growth occurs on solid media in air; colonies are 2-4 $\mathrm{mm}$ in diameter, circular, low convex with entire margins, opaque and moist. Yellowish white pigment is produced. In young cultures, small, slender rods are formed. Many cells are arranged at angles, forming $\mathrm{V}$ shapes; primary branching is uncommon. In older cultures rods are shorter, but a marked rod-coccus growth cycle does not occur. Gram-positive nonmotile rods. The optimum temperature for growth is about $28^{\circ} \mathrm{C}$, and growth occurs at $37^{\circ} \mathrm{C}$. Good growth occurs in the presence of 2, 5 and $6.5 \% \mathrm{NaCl}$. Maltose, mannose, mannitol and gluconate are assimilated, but arabinose, $\mathrm{N}$-acetylglucosamine, acetate, adipate, caprate, citrate, fumarate, lactate, malate, phenyl acetate and propionate are not assimilated. Starch, Tweens 40, 60 and 80 are hydrolysed, and gelatin and Tween 20 are hydrolysed weakly. $\mathrm{H}_{2} \mathrm{~S}$ is not produced. Methyl red test is positive and Voges-Proskauer test is negative. Arginine dihydrolase is not produced. Acid is produced from glucose, mannose and rhamnose, but is not produced from L-arabinose, galactose, inulin, melezitose, raffinose, ribose, sucrose, trehalose and xylose. The cell wall peptidoglycan contains ornithine, homoserine, glutamate plus hydroxyglutamate, glycine and alanine. The cell wall sugars are mannose, galactose and glucose. The major cellular fatty acids are anteiso-C15:0, anteiso-C17:0, iso-C15:0 and isoC16:0. Unsaturated menaquinones with 11,12 and 13 isoprene units are present. The DNA G $+\mathrm{C}$ content of the type strain is $67.2 \mathrm{~mol} \%$. Source is soil in mangrove rhizosphere. The type strain is IFO $16062^{\mathrm{T}}$ (= strain No. $76^{\mathrm{T}}$ ).

\section{Description of Microbacterium hominis sp. nov.}

Microbacterium hominis (ho'mi.nis. L. masc. n. homo man; L. gen. masc. n. hominis of man).

Good growth occurs on solid media in air ; colonies are 2-4 mm in diameter, circular, low convex with entire margins, opaque and moist. Yellowish white pigment is produced. In young cultures, small, slender rods are formed. Many cells are arranged at angles, forming $\mathrm{V}$ shapes; primary branching is uncommon. In older cultures, rods are shorter, but a marked rod-coccus growth cycle does not occur. Gram-positive nonmotile rods. The optimum temperature for growth is about $28^{\circ} \mathrm{C}$, and growth occurs at $37^{\circ} \mathrm{C}$. Growth occurs in the presence of $2 \% \mathrm{NaCl}$, but is weak in the presence of 5 and $6.5 \% \mathrm{NaCl}$. Arabinose, maltose, mannose, mannitol, $N$-acetylglucosamine, acetate, citrate, fumarate, gluconate, lactate, malate and propionate are assimilated, but adipate, caprate and phenyl acetate are not assimilated. Tweens 20, 40, 60 and 80 are hydrolysed, but gelatin and starch are not hydrolysed. $\mathrm{H}_{2} \mathrm{~S}$ is produced. Methyl red test and Voges-Proskauer test are positive. Arginine dihydrolase is not produced. Acid is produced from Larabinose, galactose, glucose, mannose, melezitose, sucrose and trehalose, but is not produced from inulin, raffinose, rhamnose, ribose and xylose. The cell wall peptidoglycan contains lysine, glutamate plus hydroxyglutamate, glycine and alanine. The cell wall sugars are rhamnose, 6-deoxy-L-talose, galactose and mannose. The major cellular fatty acids are anteiso$\mathrm{C} 15: 0$, anteiso-C17:0 and iso-C16:0. Unsaturated menaquinones with 11 and 12 isoprene units are present. The DNA G $+\mathrm{C}$ content of the type strain is $71.2 \mathrm{~mol} \%$. Source is lung aspirate. The type strain is IFO $15708^{\mathrm{T}}$.

\section{Description of Microbacterium ketosireducens sp. nov.}

Microbacterium ketosireducens [ke.to.si.redu'cens. M.L. n. ketosum keto-sugar; L. part. adj. reducens reducing (deoxidizing); M.L. part. adj. ketosireducens ketose-deoxidizing].

Good growth occurs on solid media in air; colonies are 2-4 $\mathrm{mm}$ in diameter, circular, low convex with entire margins, opaque and moist. Yellow pigment is produced. In young cultures, small, slender rods are formed. Many cells are arranged at angles, forming V shapes; primary branching is uncommon. In older cultures, rods are shorter, but a marked rod-coccus growth cycle does not occur. Gram-positive nonmotile rods. The optimum temperature for growth is about $28^{\circ} \mathrm{C}$, but growth does not occur at $37^{\circ} \mathrm{C}$. Growth occurs in the presence of $2 \% \mathrm{NaCl}$, but not in the presence of $6.5 \% \mathrm{NaCl}$. Weak growth occurs in the presence of $5 \% \mathrm{NaCl}$. Arabinose, maltose, mannose, mannitol and gluconate are assimilated, but $N$-acetylglucosamine, acetate, adipate, caprate, citrate, fumarate, lactate, malate, phenyl acetate and propionate are assimilated weakly or are not assimilated. Gelatin, starch, Tweens $20,40,60$ and 80 are hydrolysed. $\mathrm{H}_{2} \mathrm{~S}$ is produced. Methyl red test is positive and VogesProskauer test is negative. Nitrate is reduced to nitrite. Arginine dihydrolase is not produced. Acid is produced from L-arabinose, glucose, mannose, sucrose and xylose, but is not produced or is produced weakly from galactose, inulin, melezitose, raffinose, rhamnose, ribose and trehalose. The cell wall peptidoglycan contains ornithine, homoserine, glutamate plus hydroxyglutamate, glycine and alanine. The major cellular fatty acids are anteiso-C15:0, anteiso-C17:0 and iso-C16:0. Unsaturated menaquinones with 13 isoprene units are present. The DNA G $+\mathrm{C}$ content of the type strain is $69 \cdot 7-69 \cdot 8 \mathrm{~mol} \%$. Source is soil. The type strain is IFO $14548^{\mathrm{T}}$. 


\section{Description of Microbacterium maritypicum comb.} nov.

Microbacterium maritypicum (ma.ri.ty'pi.cum. L. neut. n. mare the sea; M.L. neut. adj. typicum typical; M.L. neut. adj. maritypicum typical of the sea).

Good growth occurs on solid media in air; colonies are 2-4 $\mathrm{mm}$ in diameter, circular, low convex with entire margins, opaque and moist. Light yellow pigment is produced. In young cultures, small, slender rods are formed. Many cells are arranged at angles, forming $\mathrm{V}$ shapes; primary branching is uncommon. In older cultures, rods are shorter, but a marked rod-coccus growth cycle does not occur. Gram-positive and motile rods with peritrichous flagella. The optimum temperature for growth is about $28^{\circ} \mathrm{C}$, and growth occurs at $37^{\circ} \mathrm{C}$. Growth occurs in the presence of 2 and $5 \%$ $\mathrm{NaCl}$, but is weak in the presence of $6.5 \% \mathrm{NaCl}$. Maltose, mannose, mannitol, $\mathrm{N}$-acetylglucosamine, citrate, gluconate, malate, phenyl acetate are assimilated, but arabinose, acetate, adipate, caprate, fumarate, lactate and propionate are not assimilated. Gelatin, Tweens 20, 40, 60 and 80 are hydrolysed, but starch is not hydrolysed. $\mathrm{H}_{2} \mathrm{~S}$ is not produced. Methyl red test and Voges-Proskauer test are negative. Arginine dihydrolase is not produced. Acid is produced from glucose, mannose, but is not produced or is produced weakly from L-arabinose, galactose, inulin, melezitose, raffinose, rhamnose, ribose, sucrose, trehalose and xylose. The cell wall peptidoglycan contains ornithine, homoserine, glutamate plus hydroxyglutamate, glycine and alanine. The cell wall sugar is galactose. The major cellular fatty acids are anteisoC15:0 and iso-C16:0. Unsaturated menaquinones with 12 isoprene units are present. The DNA G+C content of the type strain is $71.6 \mathrm{~mol} \%$. Source is sea water and marine mud. The type strain is IFO $15779^{\mathrm{T}}$. Flavobacterium marinotypicum (Breed, 1957) is the basonym of this species.

\section{Description of Microbacterium thalassium sp. nov.}

Microbacterium thalassium (tha.las'si.um. M.L. neut adj. thalassium based on Gr. adj. thalassios pertaining to the sea).

Good growth occurs on solid media in air; colonies are 2-4 $\mathrm{mm}$ in diameter, circular, low convex with entire margins, opaque and moist. Yellow or yellowish white pigment is produced. In young cultures, small, slender rods are formed. Many cells are arranged at angles, forming $\mathrm{V}$ shapes; primary branching is uncommon. In older cultures, rods are shorter, but a marked rod-coccus growth cycle does not occur. Grampositive non-motile rods. The optimum temperature for growth is about $28^{\circ} \mathrm{C}$, but growth does not occur at $37^{\circ} \mathrm{C}$. Growth occurs in the presence of 2 and $5 \%$ $\mathrm{NaCl}$, but is weak in the presence of $6.5 \% \mathrm{NaCl}$. Maltose, mannose, mannitol and gluconate are assimilated, but arabinose, $N$-acetylglucosamine, acetate, adipate, caprate, citrate, fumarate, lactate, malate, phenyl acetate and propionate are not assimilated. Starch, Tweens 40, 60 and 80 are hydrolysed. Gelatin and Tween 20 are hydrolysed weakly. $\mathrm{H}_{2} \mathrm{~S}$ is not produced. Methyl red test is positive and VogesProskauer test is negative. Arginine dihydrolase is not produced. Acid is produced from glucose, mannose and xylose, but is not produced from L-arabinose, galactose, inulin, melezitose, raffinose, rhamnose, ribose, sucrose and trehalose. The cell wall peptidoglycan contains ornithine, homoserine, glutamate plus hydroxyglutamate, glycine and alanine. The cell wall sugars of strain IFO $16060^{\mathrm{T}}$ are galactose and glucose, and those of strain IFO 16061 are rhamnose, mannose, galactose and glucose. The major cellular fatty acids are anteiso-C15:0, anteiso-C17:0, iso-C16:0 and/or iso-C15:0. Unsaturated mena-quinones with 11 and 12 isoprene units are present. The DNA G $+\mathrm{C}$ content of the type strain is $69 \cdot 1-69 \cdot 7 \mathrm{~mol} \%$. Source is soil in mangrove rhizosphere. The type strain is IFO $16060^{\mathrm{T}}$.

\section{REFERENCES}

Breed, R. S. (1957). Genus III. Flavobacterium Bergey et al. 1923. In Bergey's Manual of Determinative Bacteriology, 7th edn, pp. 309-322. Edited by R. S. Breeds, E. G. D. Murray \& M. R. Smith. Baltimore: Williams \& Wilkins.

Clise, E. H. (1948). Appendix to suborder Eubacteriineae. In Bergey's Manual of Determinative Bacteriology, 6th edn, pp. 692-703. Edited by R. S. Breed, E. G. D. Murray \& A. P. Hitchens. Baltimore: Williams \& Wilkins.

Collins, M. D., Jones, D., Keddie, R., Kroppenstedt, R. M. \& Schleifer, K. H. (1983). Classification of some coryneform bacteria in a new genus Aureobacterium. Syst Appl Microbiol 4, 236-252.

Collins, M. D. \& Keddie, R. M. (1986). Genus Microbacterium Orla-Jensen 1909, 337 $7^{\mathrm{AL}}$. In Bergey's Manual of Systematic Bacteriology, vol. 2, pp. 1320-1322. Edited by P. H. A. Sneath, N. S. Mair, M. E. Sharpe \& J. G. Holt. Baltimore: Williams \& Wilkins.

Cowan, S. T. (1974). Cowan and Steel's Manual for the Identification of Medical Bacteria. London: Cambridge University Press.

Ezaki, T., Hashimoto, Y. \& Yabuuchi, E. (1989). Fluorometric deoxy-ribonucleic acid-deoxyribonucleic acid hybridization in microdilution wells as an alternative to membrane filter hybridization in which radio-isotopes are used to determine genetic relatedness among bacterial strains. Int J Syst Bacteriol 39, 224-229.

Felsenstein, J. (1985). Confidence limits on phylogenies: an approach using the bootstrap. Evolution 39, 783-791.

Funke, G., Falsen, E. \& Barreau, C. (1995). Primary identification of Microbacterium spp. encountered in clinical specimens as CDC coryneform group A-4 and A-5 bacteria. J Clin Microbiol 33, 188-192.

Holmes, B. R., Owen, J. \& McMeekin, T. A. (1984). Genus Flavobacterium Bergey, Harrison, Breed, Hammer and Huntoon 1923, 97 $\mathrm{AL}$. In Bergey's Manual of Systematic Bacteriology, vol. 1, pp. 353-360. Edited by N. R. Krieg \& J. G. Holt. Baltimore: Williams \& Wilkins.

Kollöffel, B., Burri, S., Meile، L. \& Teuber, M. (1997). Development of 16S rRNA oligonucleotide probes for Brevibacterium, Micrococcus/Arthrobacter and Microbacterium/Aureo- 
bacterium used in dairy starter cultures. Syst Appl Microbiol 20, 409-417.

Larsen, N., Olsen, G. J., Maidak, B. L., McCaughey, M. J., Overbreek, R., Macke, T. J., Marsh, T. L. \& Woese, C. R. (1993). The ribosomal database project. Nucleic Acids Res 21, 3021-3023.

Mikami, H. \& Ishida, Y. (1983). Post-column fluorometric detection of reducing sugars in high-performance liquid chromatography using arginine. Bunseki Kagaku 32, E207-E210.

Saweljew, P., Kunkel, J., Feddersen, A., Baumert, M., Baehr, J., Ludwig, W., Bhakdi, S. \& Husmann, M. (1996). Case of fatal systemic infection with an Aureobacterium sp.: identification of isolate by $16 \mathrm{~S}$ rRNA gene analysis. $J$ Clin Microbiol 34, 1540-1541.

Schleifer, K. H. \& Kandler, O. (1972). Peptidoglycan types of bacterial cell walls and their taxonomic implications. Bacteriol $\operatorname{Rev}$ 36, 407-477.

Skerman, V. B. D., McGowan, V. \& Sneath, P. H. A. (1980).
Approved lists of bacterial names. Int $J$ Syst Bacteriol 30, 225-420.

Takeuchi, M. \& Hatano, K. (1998). Union of the genera Microbacterium Orla-Jensen and Aureobacterium Collins et al. in a redefined genus Microbacterium. Int $J$ Syst Bacteriol 48 , $739-747$.

Wakushima, S., Kuraishi, S. \& Sakurai, N. (1994). Soil salinity and $\mathrm{pH}$ in Japanese mangrove forests and growth of cultivated mangrove plants in different soil conditions. $J$ Plant Res 107, 39-46.

Yokota, A., Takeuchi, M., Sakane, T. \& Weiss, N. (1993a). Proposal of six new species in the genus Aureobacterium and transfer of Flavobacterium esteraromaticum Omelianski to the genus Aureobacterium as Aureobacterium esteraromaticum comb. nov. Int $J$ Syst bacteriol 34, 555-564.

Yokota, A., Takeuchi, M. \& Weiss, N. (1993b). Proposal of two new species in the genus Microbacterium: Microbacterium dextranolyticum sp. nov. and Microbacterium aurum sp. nov. Int $J$ Syst Bacteriol 43, 549-554. 